\title{
Kritische Kooperationen erwünscht
}

\section{Muslimische Vereine als mögliche Partner in der Sozialen Arbeit}

\author{
Alp Otman
}

Alp Otman ist seit zehn Jahren Leiter des Interkulturellen Büros der Stadt Darmstadt, davor war er stellvertretender Leiter des Amtes für multikulturelle Angelegenheiten in Frankfurt am Main.

E-Mail interkulturell@darmstadt.de
Die wichtigste, weil strukturelle Dimension der Integration, als eines komplexen und wechselseitigen Prozesses, ist die gleichberechtigte Eingliederung der Migrantinnen und Migranten in die Teilsysteme der Aufnahmegesellschaft. Auch soziale Dienste und Einrichtungen müssen sich dieser Aufgabe stellen.

Migrantinnen und Migranten entwickeln in den Zuwanderungsländern engere Kontakte innerhalb ihrer eigenen Sprachgruppe und bilden Netzwerke mit Funktionen wie Selbsthilfe, Stabilisierung der Persönlichkeit, community-spezifische Sozialisation, soziale Kontrolle und Interessenvertretung. (1) Die Community-Bildung ist ein regelmäßiges Phänomen in der Anfangsphase jeglicher Migration und kann im weiteren Verlauf unter Einwirkung von inneren und äußeren Faktoren zur stärkeren Öffnung oder Abschottung führen. Selbstorganisationen von Zugewanderten - die Migrantenvereine gehören zu den wichtigen Strukturelementen der Community-Bildung (vgl. Heckmann 2001, S. 17 ff.).

Der Integrationsprozess, der ohnehin nur zu einem bestimmten Grad gesteuert werden kann, ist um so erfolgreicher, je stärker die Ressourcen der MigrantenCommunities für die Öffnung zur Aufnahmegesellschaft genutzt werden. Deshalb ist die Partizipation von Migrantinnen und Migranten (nicht nur aber auch mittels ihrer Vereine) ein wichtiger Faktor für die Effektivität und Nachhaltigkeit der Integrationspolitik.

Es gibt unterschiedliche Typen der Migrantenvereine, je nachdem ob kulturelle, politische, religiöse, soziale oder wirtschaftliche Aspekte überwiegen. Unter ihren Tätigkeiten hat die Soziale Arbeit von Beginn an einen wichtigen Raum eingenommen. Da sie aber immer auch als Instrument der Community-Bildung fungiert, kann sie sich genauso ambivalent auswirken - in Richtung einer Öffnung oder Abschottung (vgl. Gaitanides 2003, S.27).
Seit Beginn der 1990er Jahre besteht bei einigen säkularen Migrantenvereinen, die sich der Sozialen Arbeit zuwenden, eine Tendenz zur Teilprofessionalisierung. Professionelle Vertreterinnen und Vertreter der Vereine können Vermittlungsaufgaben zwischen MigrantenCommunities und Institutionen der Aufnahmegesellschaft übernehmen, wenn die Soziale Arbeit auf Öffnung orientiert ist. (2)

\section{Entstehung und Entwicklung muslimischer Vereine und Verbände in Deutschland}

Die meisten muslimischen Vereine in Deutschland - größtenteils türkischer und marokkanischer Herkunft - sind ursprünglich als multifunktionale Selbstorganisationen gegründet worden. Mit der Zeit entwickelten sie sich zu Moscheevereinen, wobei ihre ursprüngliche Multifunktionalität oft in abgeschwächter Form erhalten blieb. Die Entwicklung der Bindungen zu den Herkunftsländern, aber auch zu finanzstarken Ölländern, allen voran zu Saudi-Arabien, und die Unterstützung durch sie veranlasste die Gründung von immer mehr muslimischen Vereinen (1970er und 1980er Jahre). Konkurrenz um mehr Einfluss auf die Gläubigen und Richtungskämpfe führten zu Fraktionierungen, aber auch zur Bildung von Verbänden auf Bundes- und auf europäischer Ebene (1980er und 1990er Jahre). Seit Mitte der 1990er Jahre verlagerte sich diese Dynamik auf die Institutionalisierung mit der Forderung zur Anerkennung als Religionsgemeinschaft - es kam zur Gründung von Zweckbündnissen auf Landes- oder Bundesebene (vgl. Kasten: »Muslimische Verbände in Deutschland « - Literatur siehe Anmerkung 3).

Die formellen Vereinsstrukturen sind nicht die einzige Form der Organisierung der muslimischen Migrantinnen und Mi- 
granten in Deutschland. Mindestens genauso wichtig, wenn nicht wichtiger, sind die Ordensgemeinschaften (vgl. SpulerStegemann, S. 127 ff.), allen voran der mächtige Nakschibendiye-Orden mit seinen vielen Abzweigungen wie die »Süleyman-Anhänger « (die als Ausnahmeerscheinung innerhalb dieser Gruppe die Bildung des Verbands der Islamischen Kulturzentren e. V. - VIKZ - betrieben haben) oder »Nurculuk-Anhänger « und als jüngste Abzweigung davon die wohl potenteste »Fethullah-Anhänger «, um nur die größten zu nennen. (4) Zahlenmäßig nicht so groß, aber von der extremen Radikalität des Islamverständnisses her wichtig, sind die »Kaplan-Anhänger «. Das Besondere dieser sufitischen Ordensgemeinschaften besteht $u$. a. in der »verdeckten " Organisationsform, die es ihnen ermöglicht, sich unbemerkt innerhalb der Vereinsbasis anderer Richtungen zu organisieren oder aber in Vereinen mit unverfänglich klingenden Namen.

Diese komplexe Gesamtstruktur macht es oft außerordentlich schwierig, zu einer richtigen Einschätzung des betreffenden Vereins zu gelangen. Nach übereinstimmenden Schätzungen von vielen Expertinnen und Experten übersteigt aber die Anzahl der in Vereinen organisierten Musliminnen und Muslime nicht 15 Prozent aller hier lebenden Menschen muslimischen Glaubens, die Mehrheit dieses Bevölkerungsteils ist also nicht in solchen Vereinigungen organisiert.

Der Vollständigkeit halber sei erwähnt, dass es neben diesen organisatorischen Strukturen eine regionale »Initiative von säkularen und laizistischen Bürgerinnen und Bürgern aus islamisch geprägten Herkunftsländern in Hessen « (ISL Hessen) existiert, die die Stimme der »schweigenden Mehrheit « sein will und säkulare und laizistische Positionen einnimmt. (5)

\section{Muslimische Vereine kaum mit deutschen Religionsgemein- schaften oder Wohlfahrts- verbänden vergleichbar}

Die Religionsgemeinschaft wird im deutschen Staatskirchenrecht als demokratische Kooperationspartnerin des Staates verstanden, die durch ihre verfasste hierarchische Struktur die Gewähr für die dauerhafte Repräsentanz ihrer Gemeinschaft geben kann. Dagegen kennt der sunnitische Islam in seiner Geschichte keine Repräsentanz und keine verfasste hierarchische Struktur, und dieser Unterschied ist nicht zufälliger sondern systematischer Natur, weil die Ablehnung des Klerus eine wichtige Begründung in der Entstehung des Islam darstellt.
Dass auch die muslimischen Vereine ehrenamtlich Soziale Arbeit leisten, ist selbstverständlich. Dabei wird der Charakter der Sozialen Arbeit mehr oder weniger stark von den Gesamtinteressen der Vereine (z. B. Einflusserweiterung, Anerkennung in der Öffentlichkeit, religiöse

\section{»Angebote zur Kooperation müssen im Einzelfall entschieden werden"}

Der Anspruch der sunnitischen Verbände in Deutschland auf Anerkennung als Religionsgemeinschaft ist daher nicht aus dem Islam ableitbar, sondern nur vor dem Hintergrund der deutschen Gesetzgebung verständlich. Die Verbände möchten eine formale Gleichstellung mit den Kirchen erlangen und die materiellen und ideellen Vorteile einer Religionsgemeinschaft (langfristig einer Körperschaft des öffentlichen Rechts) genießen.

\begin{abstract}
Missionierung, community-spezifische Sozialisation und soziale Kontrolle, Lobby-Arbeit für die Herkunftsländer) geprägt. Unter den muslimischen Verbänden hat die Islamische Gemeinschaft Milli Görüs (IGMG) ihre Soziale Arbeit im Frauen- und Jugendbereich im Verhältnis $\mathrm{zu}$ anderen intensiv entwickelt und versucht hartnäckig, Kommunen und Wohlfahrtsverbände für eine Kooperation zu gewinnen. Die Frage ist allerdings, ob sie demokratische Krite-
\end{abstract}

\section{Muslimische Verbände in Deutschland}

Einer der wichtigsten muslimischen Verbände in Deutschland ist die Türkisch-Islamische Union der Anstalt für Religion e. V. (DITIB), die, wenn nicht formell so de facto, der Behörde für Religionsangelegenheiten in der Türkei untersteht. Nach Schiffauer findet sich eine »individuierte « Position zum Islam eher innerhalb der DITIB-Vereine, deren Islam-Verständnis als privates Verhältnis zwischen Mensch und Gott definieren lässt, auch wenn das Potenzial der zweiten Generation für die Entwicklung eines liberalen Islam bei weitem nicht ausgeschöpft wird (vgl. Schiffauer 2004, S. 364).

Die »Islamische Gemeinschaft Milli Görüs « (IGMG) ist der größte Einzelverband im Islamrat und wird regelmäßig kritisch im Verfassungsschutzbericht aufgeführt. Nach Schiffauer dominiert nach einem Führungswechsel Mitte der 1990er Jahre eine »kommunalistisch-orthodoxe " Position in ihr, deren Bestrebungen, die »ultraorthodoxe « Position innerhalb der älteren Generation zurückzudrängen, über Öffnung oder Abschottung des Verbands entscheiden wird (vgl. ebenda). Das Problem für die »Orthodoxen « ist nach Schiffauer, das Verhältnis von Scharia und Leben in der nicht-islamischen Gesellschaft zusammen zu denken. Kritisch anzumerken bleibt allerdings, dass ihre Position nur dann interessant wäre, wenn sie sich von der "Schariaisierung « der Moderne distanziert, die gerade das Kampfprogramm der Islamisten ist.

Schließlich spielt auch der Zentralrat der Muslime in Deutschland (ZMD) eine Rolle. Viele seiner Einzelorganisationen werden ebenfalls im Zusammenhang mit der Muslimbruderschaft kritisch im Verfassungsschutzbericht erwähnt.

Die Föderation der Aleviten-Gemeinden in Deutschland (AABF) (vgl. Spuler-Stegemann, S.36 ff.) ist die Organisationsform einer differenten Glaubensrichtung innerhalb des Islam, die mit den zuvor erwähnten sunnitischen Verbänden (aber auch mit schiitischen) nicht vergleichbar ist und deren demokratische Haltung zur Verfassung nicht in Frage steht. Die kontroverse Diskussion innerhalb der alevitischen Gemeinden, ob ihr Glauben überhaupt innerhalb des Islam angesiedelt ist oder eine eigenständige Religion darstellt, ist nicht abgeschlossen.

Alp Otman 
rien und professionelle Standards zu erfüllen vermag.

\section{Kriterien der Kooperation}

Die Frage nach den Möglichkeiten und Grenzen der Kooperation mit muslimischen Vereinen hängt zunächst von den Zielen ab. Geht es um die Betreuung einer einzelnen Familie? Geht es darum, mit den Eltern einer Kindereinrichtung ins Gespräch zu kommen? Oder handelt es sich um ein präventives Projekt, mit dem möglichst viele Mitglieder des Vereins erreicht werden sollen?

Das zweite Kriterium betrifft die Rolle, die dem muslimischen Verein bei der Kooperation zugedacht ist. Handelt es sich um eine eher passive Unterstützung, oder soll der Verein selbstständig als Akteur der Sozialen Arbeit auftreten?

Schließlich werden die Möglichkeiten und Grenzen der Kooperation davon abhängen, wie weit das Leitbild, die Ziele und Methoden der Sozialen Arbeit bei den Kooperationspartnerinnen und Kooperationspartnern übereinstimmen. Erkenntnisse über den Verband und seine Bindungen zum Ausland sind dabei genauso unerlässlich wie Informationen über den konkreten Ortsverein.

Säkulare Arbeitsgespräche mit Vereinen über eine mögliche Kooperation sind $\mathrm{zu}$ unterscheiden von Veranstaltungen, die unter dem Titel »interreligiöser Dia$\log$ « laufen. Während letztere in der Regel diplomatische Good-will-Begegnungen auf repräsentativer Ebene sind, sollten erstere offene, problemorientierte und kritische Diskussionen einschließen.

Kooperationen mit Basisvereinen aus Verbänden, die nicht uneingeschränkt für das Grundgesetz eintreten, sind genauso abzulehnen, wie mit einem Basisverein, der zwar einem unproblematischen Verband angehört, aber in dessen Reihen vor Ort ein patriarchalisches Verständnis der Sozialen Arbeit oder antisemitische Vorurteile dominieren. Entsprechend der Einschätzung des lokalen Vereins und seines Verbands können daher Kooperationen mit unterschiedlicher Art, Intensität und Reichweite entstehen - oder auch nicht.

Eine sinnvolle Alternative zu der Kooperation mit muslimischen Vereinen kann der Einsatz von zweisprachigen Multiplikatorenkräften muslimischer Herkunft sein, die gute Kontakte zu der Basis dieser
Vereine haben, ohne unbedingt bei ihnen Mitglieder zu sein. Dafür sollten sie eine bestimmte Weiterbildung durchlaufen haben. (6) Für Kontakte zu einzelnen Familien, für Elterngesprächskreise in einem Kindergarten oder für ein präventives Projekt mit muslimischen Migrantinnen und Migranten wurden mit weiterqualifizierten Assistenzkräften gute Erfolge erzielt.

\section{Anmerkungen}

(1) Für eine ausführlichere Erläuterung vgl. Otman, 2007, S. 19 ff.

(2) Exemplarisch für die kommunale Förderung der Selbsthilfepotenziale vgl. Latorre, 2005, S.299 ff.

\section{Literatur}

Gaitanides, Stefan (2003): Selbsthilfepotenzial von Familien ausländischer Herkunft, freiwilliges Engagement und Selbstorganisationen von Migranten Struktur, Funktion, Förder- und Anerkennungsdefizite, in: Migration und Soziale Arbeit, Heft 2, Frankfurt am Main.

Heckmann, Friedrich (2001): Ethnische Kolonien: Schonraum für Integration oder Verstärker der Ausgrenzung, in: Interkulturelles Büro Darmstadt (Hg.): Öffnung der Migranten-Communities, Darmstadt.

Latorre, Patricia (2005): Förderung statt Fürsorge, Ansätze, Formen und Instrumente der Stärkung der Eigenressourcen von Migranten in der Kommune, in: Treischler, Andreas und Cyrus, Norbert (Hg.): Handbuch soziale Arbeit in der Einwanderungsgesellschaft, Frankfurt am Main.

Otman, Alp (2007): Migrantenfamilie und Community zwischen Öffnung und Abschottung, in: Mansfeld, Cornelia (Hg.): An den Stärken ansetzen. Interkulturelle Eltern- und Familienbildung, Arbeitspapiere aus der EFH, Darmstadt, Internet http://web.efhd.de/ Arbeitspapier_Nr.9.pdf

Schiffauer, Werner (2004): Vom Exilzum Diaspora-Islam. Muslimische Identitäten in Europa, in: Soziale Welt, Heft 4.

Spuler-Stegemann, Ursula (2002): Muslime in Deutschland. Informationen und Klärungen, Freiburg im Breisgau.
(3) Für einen umfassenden Überblick vgl. Spuler-Stegemann, 2002, S.101 ff.

(4) Derzeit expandieren die FethullahAnhänger in Deutschland mit eigenen Schulen und Nachhilfe-Einrichtungen unter verschiedenen Namen.

(5) Vgl. Internet http://www.isl-hessen.de

(6) Vgl. Internet http://www.equal.ikbdarmstadt.de

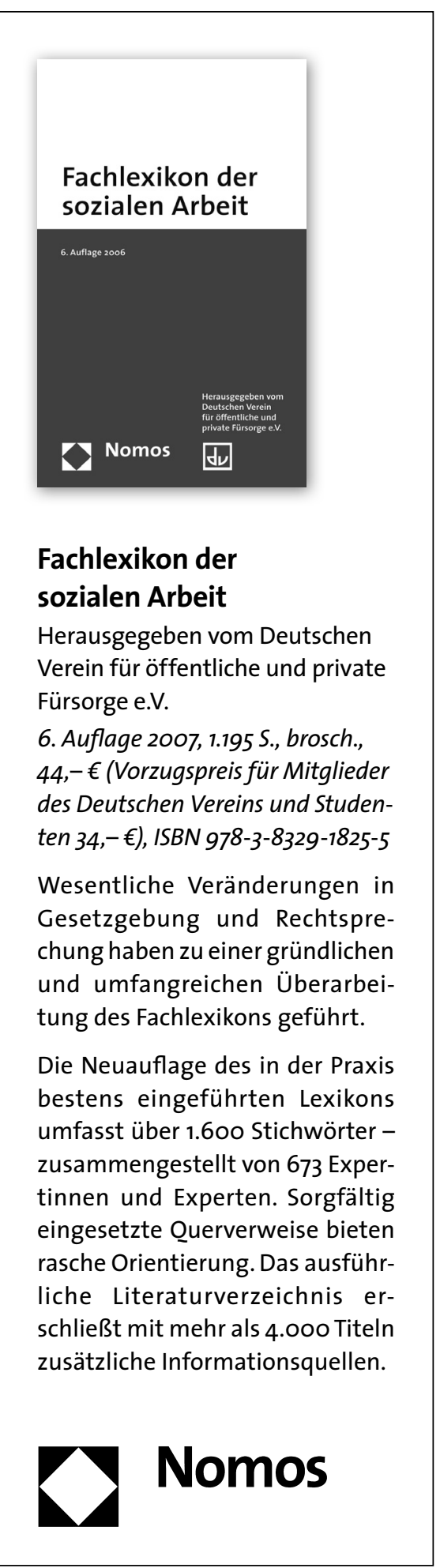

